\title{
Multi-purpose Phantom Realization for Quality Controls of MRI System
}

\author{
Hatem Besbes ${ }^{1}$, Hossam Donya ${ }^{1,2, *}$ \\ ${ }^{1}$ Medical Physics Division, Physics Department, Faculty of Science, King Abdulaziz University, Jeddah, Saudi Arabia \\ ${ }^{2}$ Physics Department, Faculty of Science, Menoufia University, Shebin El-Koom, Egypt
}

Email address:

donyavip@yahoo.com (H. Donya),hdunia@kau.edu.sa (H. Donya),

${ }^{*}$ Corresponding author

\section{To cite this article:}

Hatem Besbes, Hossam Donya. Multi-purpose Phantom Realization for Quality Controls of MRI System. European Journal of Clinical and Biomedical Sciences. Vol. 3, No. 3, 2017, pp. 59-66. doi: 10.11648/j.ejcbs.20170303.12

Received: October 13, 2016; Accepted: March 1, 2017; Published: June 5, 2017

\begin{abstract}
Searching for a multi-purpose phantoms (MTPs) is a key issue for quality control (QC) in magnetic resonance imaging (MRI) technique. This main objective of this article is to design MTP phantom for MRI quality control/assurance of relatively low cost components. Our proposed phantom costs only around $23 \%$ out of the price of American College of Radiology (ACR) phantom. A good choice of MTP polyamide material and manufacturing procedures leads to a realization of an adequate phantom at an affordable cost, with more options and easy to maintain compared to the available ACR phantoms. A comprehensive QC is investigated on MTP for MRI system. This study also shows the various parameters to be controlled by accreditations as stated by ACR for our proposed MTP.
\end{abstract}

Keywords: Quality Control (QC), Multi-purpose Phantom (MTP), American College of Radiology, Magnetic Resonance Imaging

\section{Introduction}

MRI becomes a main pillar of medical imaging during last twenty years. Constructed MRI images are depending on the magnetic properties of the human body. It consists of recorded signals coming from energy exchanged with nuclear magnetic moments of hydrogen nucleus in the tissue/organ. MRI is even considered as a reference in terms of precision and high quality of medical images. This property is acquired by the multitude of contrast parameters like longitudinal relaxation time $\left(\mathrm{T}_{1}\right)$, transversal relaxation time $\left(\mathrm{T}_{\mathrm{t}}\right)$ and spin density $\rho[1]$. As for all medical imaging modalities and for optimal operating, MRI machines must undergo quality control (QC) protocols. These protocols are usually made through acquisitions on an American College of Radiology (ACR) phantom as reported by Chien-Chuan et al. [2]. The current work is a continuing effort for establishing technical phantoms to be used in different images modalities [3]. This paper presents a novel methods of fabrication multi-purpose phantom (MTP) to offer more flexibility and new options for a remarkably low cost components. QC protocols in MRI are taken each one individually. Current research on MTP is focused on designing different patterns of the phantom while respecting the dimensions of the MRI antenna (place of housing the phantom during image acquisition).

\section{Material and Methods}

We start by designing MTP in our laboratory workshop from polyamide based material. For manufacturing phantom parts (See Figure 1), the machining method used in the working is milling machine. Our newly designed phantom is filled with an aqueous solution, when assembling the various components. It is necessary to pay attention to the waterproofing of the structure. For this reason, silicon and recessed cylinder are used (See Figure 2). Figure 3 shows the phantom assembly through the stage of fixing some fabricated components 


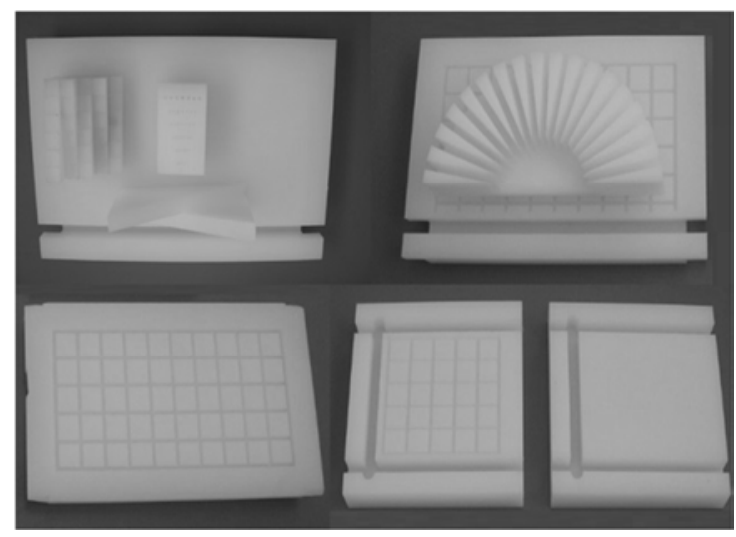

Figure 1. Established different phantom components of final designed shapes.

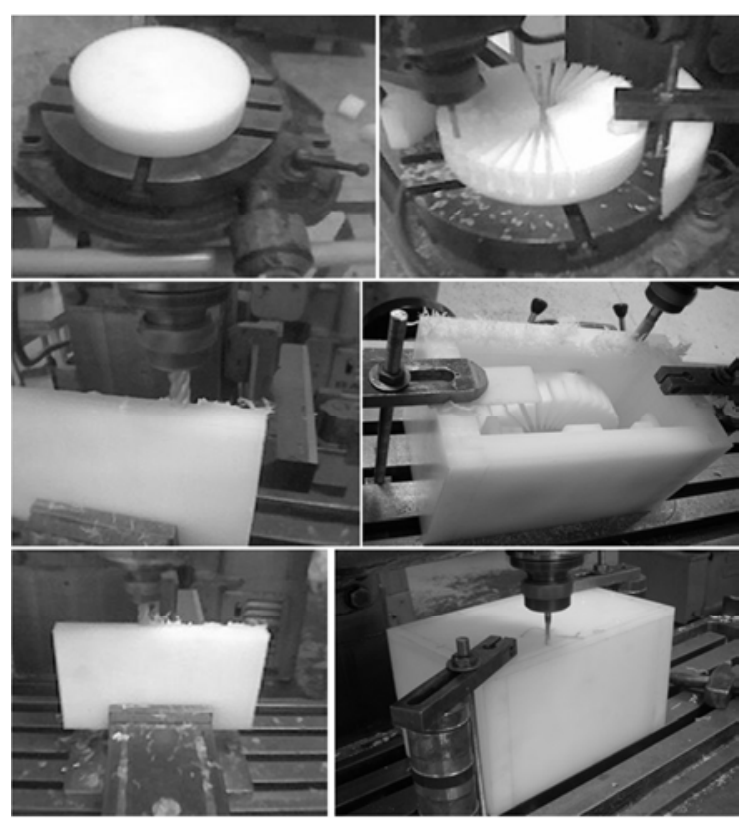

Figure 2. Milling of some phantom components.

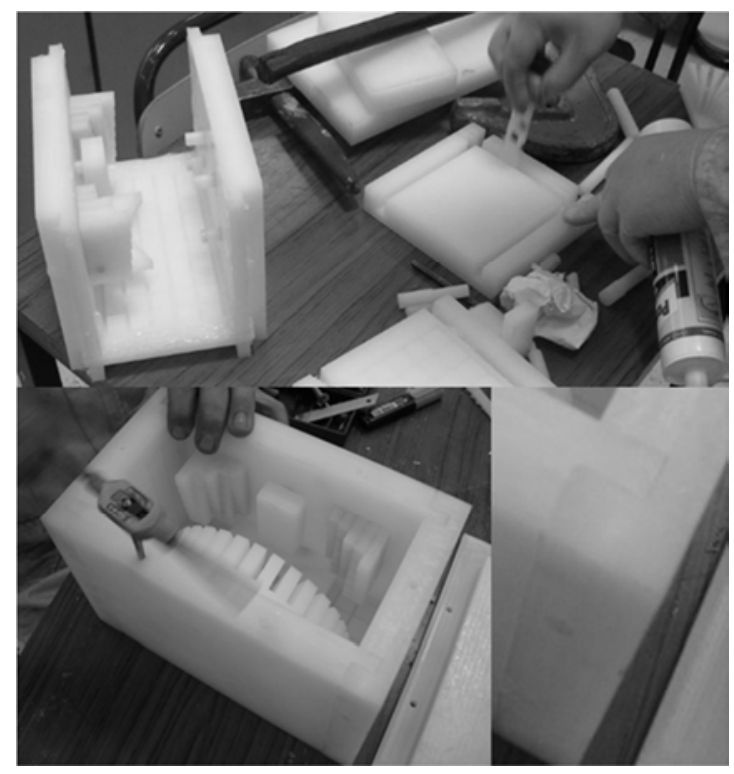

Figure 3. Phantom assembly.

\subsection{QC for Magnetic Resonance Imaging System}

QC is the assembly of systematic planned actions to improve or maintain the performances of a system. The system protocols of QC guarantee the acquisition of images, which describe in a more or less accurate way the explored internal anatomical structure. This is achieved by controlling the following parameters:- image uniformity, central resonance frequency $\left(f_{0}\right)$, slice thickness, spatial resolution, contrast, Spatial Linearity (SL), Signal-to-Noise ratio $(\mathrm{S} / \mathrm{N})$, gradient field linearity, slice position and separation in addition to relaxation times $T_{1}$ and $T_{t}$. QC is realised by image acquisitions on suitable phantoms which occupied with solution comprising a contrast product as $\mathrm{CuSO}_{4}$ and $\mathrm{MnCl}_{2}$. Concept of phantom material choice takes into account the constancies of both heat and chemistry properties against the concentrations related to human tissue protons [3].

\subsubsection{Central Resonance Frequency $\left(f_{o}\right)$}

$\mathrm{f}_{0}$ is RF which counterparts the static $\mathrm{B}_{0}$-field as stated by Larmor equation (1) [1]:

$$
f_{0}=\frac{\gamma}{2 \pi} \mathrm{B}_{0}
$$

where $\gamma$ is the charged particle magnetogyric ratio which have Hertz/tesla unit. Central resonance frequency produces a uniform cylindrical signal in MTP phantom, which is used most often for checks. Same MTP is also used for S/N and uniformity characterization. Phantom is put on the magnet centre and frequency is accustomed to regulating $\mathrm{f}_{0}$ synthesizers to accomplish high frequency [3, 4].

\subsubsection{Signal- to- Noise Ratio ( $\mathrm{S} / \mathrm{N})$}

$\mathrm{S} / \mathrm{N}$ is an essential parameter used for imaging systems characterisation. The average value of ROI (range of interest) pixel excluded the offset is considered our working signal (S). Noise is defined as the unsystematic discrepancy in intensity $[3,5,6,7]$. The measured signal using ROI should contain at least ten percentages of the signal area produced by the material, each is large. The range must be placed at the image centre and had better not take in any apparent objects. The resulting $\sigma$ (standard deviation) from unchanged ROI is defined as noise level. So, $\mathrm{S} / \mathrm{N}$ is defined as the ratio:

$$
S N R=\frac{S}{\sigma}
$$

Really, it has been found that $\mathrm{S} / \mathrm{N}$ of $95: 1$ or greater yields an acceptable results [2] in terms of acquired image quality for medical diagnosis purposes.

\subsubsection{Image Uniformity}

Ability of MRI system to get a stable signal response all over the covered volume is referred to image uniformity, when the imaged object has homogeneous MRI characteristics. Phantom that characterises image uniformity evaluation is similar to that used for signal-to-noise 
determination.

$\mathrm{S}_{\max }$ and $\mathrm{S}_{\min }$ (higher and lower signal values respectively) are developed for the pixel area at the phantom centre geometry that cover nearly $75 \%$ of its area. Edge of artefacts must be excluded from the required range $[3,6,8,9] . \Delta$ and $\overline{\mathrm{S}}$ are defined as follows:

$$
\Delta=\frac{S_{\max }-S_{\min }}{2} ; \bar{S}=\frac{S_{\max }+S_{\min }}{2}
$$

Finally, the relationship for calculating integral uniformity $\left(\mathrm{U}_{\Sigma}\right)$ is given by:

$$
U_{\Sigma}=\left[1-\frac{\Delta}{\bar{S}}\right] \cdot 100 \%=\left[1-\frac{S_{\max }-S_{\min }}{S_{\max }+S_{\min }}\right] \cdot 100 \%
$$

Good integral uniformity using equation (4) is achieved when $\mathrm{U}_{\Sigma}$ equal $100 \%$. Integral uniformity must be usually eighty percent (more than $20 \mathrm{~cm}$ in a view field or fewer).

\subsubsection{Spatial Linearity ( $S L)$}

SL scales the distortion in image geometry induced by some imaging system. For measuring SL, phantom must subjugate at least sixty percent through main view of the field that contains consistent objects array of a determined dimension and spacing. Phantom must be occupied with solution that helps in producing a signal [3]. Inconsistency of data is clearly distinguished from large scale. Thus, percentage in distortion (PD) is defined as follows:

$$
P D=\frac{|R D-O D|}{R D} \cdot 100 \%
$$

where $\mathrm{RD}$ and $\mathrm{OD}$ are real and observed dimensions, respectively. Distortion percentages of the SL are generally considered as acceptable if they are less than $5 \%$.

\subsubsection{Spatial Resolution of Maximum Contrast}

Spatial resolution of maximum-contrast is the ability of the system to distinguish objects after subtraction of noise elements. It is also greatly dependant of size of the pixel through view filed matrices [3].

Visual evaluation of spatial resolution of maximumcontrast is also depending on arrays of bars or rods. Patterns comprise of alternative signal and non-signal producing isolated areas by widths of rod or bar from the neighbours. Patterns resolution of square bar may be related to line pairs per $1 \mathrm{~mm}$ in the smallest resolvable array element. The better resolvable image analysis is evaluated by the most distinguished element in the array.

High contrast resolution remains constant under the same scan conditions for repeated measurements [3].

\subsubsection{Slice Thickness (ST)}

Slice thickness (ST) characterizes full width at halfmaximum (FWHM) of a slice outline $[3,10,11]$. ST can be evaluated by different phantoms, lots of them use variant of an inclined surface. HSR (high signal ramps) consists of inverse pairs of ramp directed at a certain $\theta$ (see Figure 1). Signal level is recorded through the ramp along the line directed orthogonally to its dimension (Figure 4). For better $\mathrm{S} / \mathrm{N}$, it is advised to working on a lot of line profiles and FWHM have to be defined for correlated ramps [3]. FWHM values is given by:

$$
F W H M(a, b, \theta)=\frac{(a+b) \cos \theta+\sqrt{(a+b)^{2} \cos ^{2} \theta+4 a b \sin ^{2} \theta}}{2 \sin \theta}
$$

The FWHM intensity profile of the first and second ramps (ramp 1 and ramp 2) refer to $a$ and $b$, respectively (Figure 4). When $\theta=90^{\circ}$, equation (5) can be written [8] as:

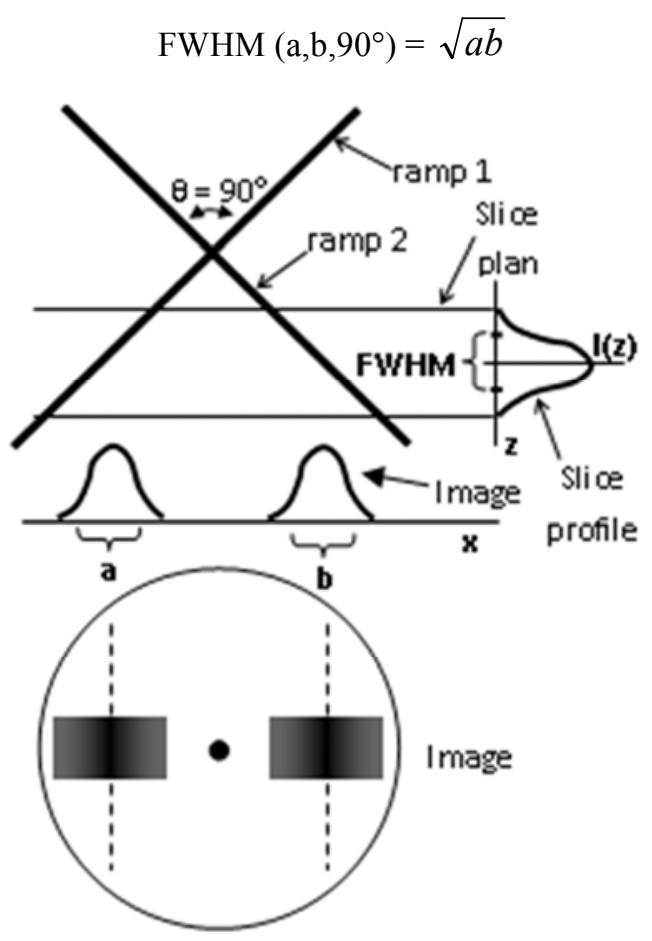

Figure 4. Calculation of ST with acquisitions done on $90^{\circ} \mathrm{HSR}$ phantom.

\subsubsection{Slice Position/Separation}

The distance between any 2 slice positions is termed slice separation $(S)$. There is outer device for specification of slice positions [3]. Generally, one phantom may be used for characterizing this tool and in addition to ST measurements as shown in Figure 4. FWHM midpoint of the profile of image slice is first prescribed (see Figure 5). $S$ from the centre of the profile to a landmark that unchanged from sliceto-slice is measured and associated with the slice position $(N)$. The relation between $N$ and $S$ of slices depends on $\theta$ between the slices is determined via equation (6):

$$
N=\frac{S}{\tan (\theta / 2)}
$$



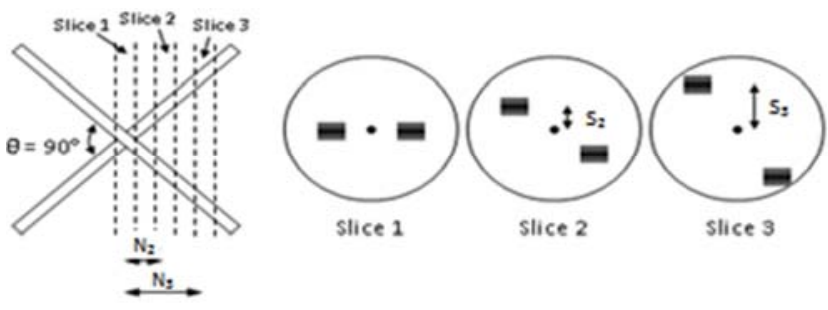

Figure 5. Phantom of the slice-thickness evaluation.

The precision in determining $N$ must be within plus or negative two millimetre $[3,10]$. On the other side, $S$ deviation from the ideal value may be less than one fifth of the total $S$ or plus or negative one $\mathrm{mm}$, (larger one is considered [3]).

\subsection{Phantom Realisation}

\subsubsection{Materials of the Phantom}

For choosing constructed filler materials of phantoms for QC, physical and chemical properties should be considered. So the stability of both thermal and chemical for these material must be checked carefully. Also, fitting parameters of $T_{1}, T_{t}$ and $\rho_{p}$ (density values of protons) are aimed to be inside range of the biological system. The material should not have any other additive magnetic susceptibility different than filling one. The recommended conditions for the selected phantom material to fulfil the following features $[3,12]$ :

$$
\begin{gathered}
100 \mathrm{~ms}<\mathrm{T}_{1}<1200 \mathrm{~ms} \\
50 \mathrm{~ms}<\mathrm{T}_{\mathrm{t}}<400 \mathrm{~ms} \\
\rho \approx \text { Density of water }
\end{gathered}
$$

Polyamide is considered as a filling material of the proposed MTP phantom (See Figure 6). Phantom is occupied with $20 \mathrm{mM}$ of $\mathrm{CuSO}_{4}$ for magnetic resonance imaging acquisitions.

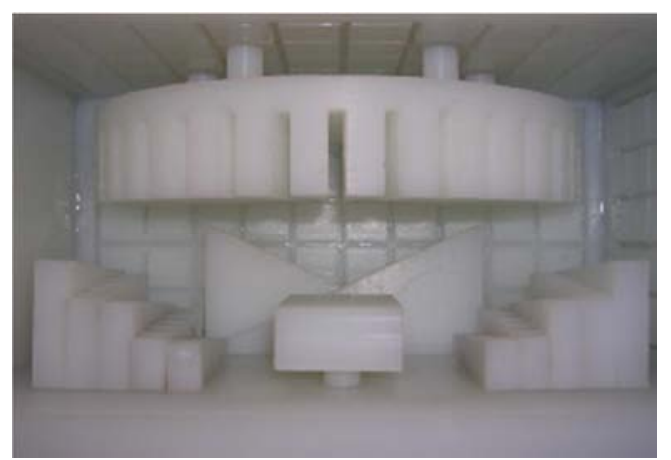

Figure 6. MTP phantom composed of - SL patterns, ST and N patterns, uniformity, $S / N$ and $f_{o}$ and other patterns.

\subsubsection{Spatial Linearity Pattern (SLP)}

SLP is a tool to measure the geometrical distortions all space directions. It involves crossed linear grooves performed on tree orthogonal faces (See Figure 7-a).

\subsubsection{Position and ST Patterns}

2 stairs ramps of circular shapes are created, each, of 30 rallies of $1 \mathrm{~mm}$ and circulated on 5 rows of 6 marches (see Figure 7-b). It helps to regulate longitudinal ST, angular direction. It is comprising of mined hollows in semi disk of width equalled five millimetres of separation angle of ten degree (see Figure 7-b). It allows to confirm the transversal and coronal ST, separation and position.

\subsubsection{Gradient Uniformity Pattern}

It contains 2 reverse triangles of dimension $100 \mathrm{~mm} \times 50$ $\mathrm{mm}$ (see Figure 7-d) which allows gradient uniformity the valuation.

\subsubsection{Uniformity, $S / N$ and $f_{o}$ Pattern}

Three criteria of uniformity, $\mathrm{S} / \mathrm{N}$ and $\mathrm{f}_{\mathrm{o}}$ are evaluated fully in cylindrical phantom of a uniform signal. It should be measured on the phantom quota that doesn't contain any other patterns.

\section{Results and Discussion}

Measurements are performed on a 1.5T MRI machine. On this machine, all acceptance tests are done. Acquisition type of spin echo is made by ST of $3 \mathrm{~mm}$ realized on Siemens $1.5 \mathrm{~T}$ machine that quantitative and qualitative explanations are based on it. This implementation is made to have a reasonable images of good $\rho_{\mathrm{p}}$ distribution.

\subsection{Uniformity and $S / N$}

For the uniformity test, it is considered the large region of interest (ROI) (Figure 8) for an acquisition done on the homogeneous phantom's longitudinal section (for more precision, we can do the same thing in the transversal and coronal directions). For the calculation of uniformity parameter (Table1), we determine the minimal $\left(\mathrm{S}_{\min }\right)$ and maximal $\left(\mathrm{S}_{\max }\right)$ values of pixels grey levels in this ROI. For the calculation of the $\mathrm{S} / \mathrm{N}$ (Table 1), Small ROI in the same acquisition is considered where the artefacts are avoided.

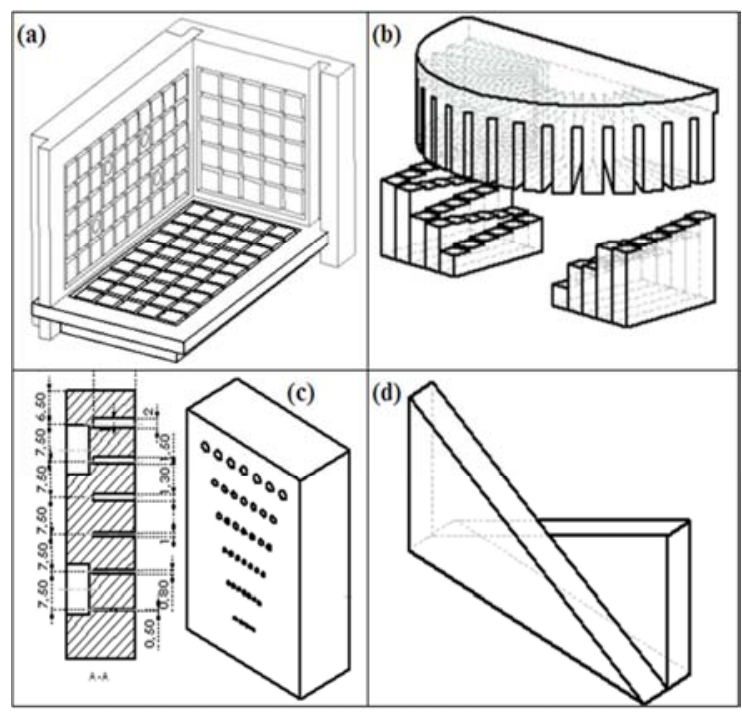

Figure 7. Patterns of the MTP phantom (a: SL (X, Y and Z), b: Slice thickness, position and separation, $c$ : Spatial resolution, $d$ : gradient uniformity). 
Table 1. Uniformity and $S / N S_{\min }$, Standard partial molar enthalpy $h_{t}^{\circ}$

\begin{tabular}{llll}
\hline Uniformity & & $\mathbf{S} / \mathbf{N}$ & \\
\hline $\mathbf{S}_{\min }$ & $\mathbf{S}_{\max }$ & $\mathbf{S}_{\min }$ & $\mathbf{S}_{\max }$ \\
\hline 146 & 175 & 146 & 175 \\
$\mathrm{U} \Sigma$ & $\mathrm{S} / \mathrm{N}$ & \\
$90.3 \%$ & & $104: 1$ & \\
\hline
\end{tabular}

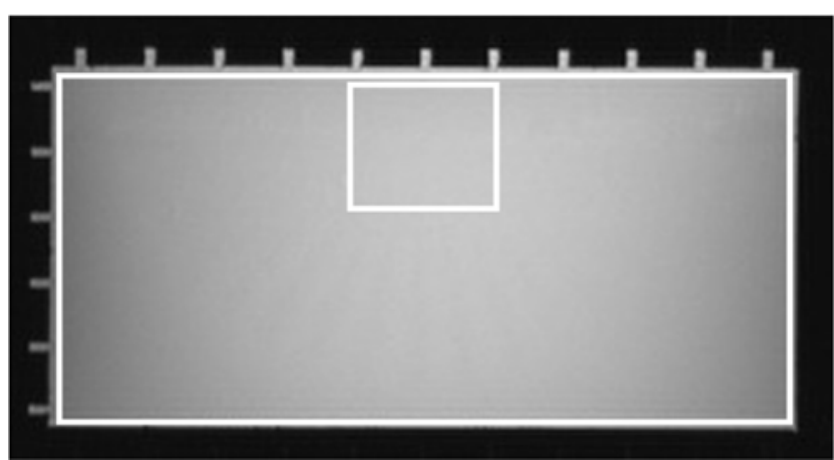

Figure 8. Uniformity and signal to noise ratio tests.

Table 2. Spatial distortion percentage.

\begin{tabular}{lll}
\hline Direction & Longitudinal & transversal \\
\hline Distortion percentage & $0.53 \%$ & $0.54 \%$ \\
\hline
\end{tabular}

\subsection{Spatial Linearity (SL)}

For the linearity tests, the acquisitions (Figure 9) are conducted at the level of basis and to lateral faces of the phantom (Figure 9). The percentages of spatial distortion are calculated with equation (3) and presented in Table 2.
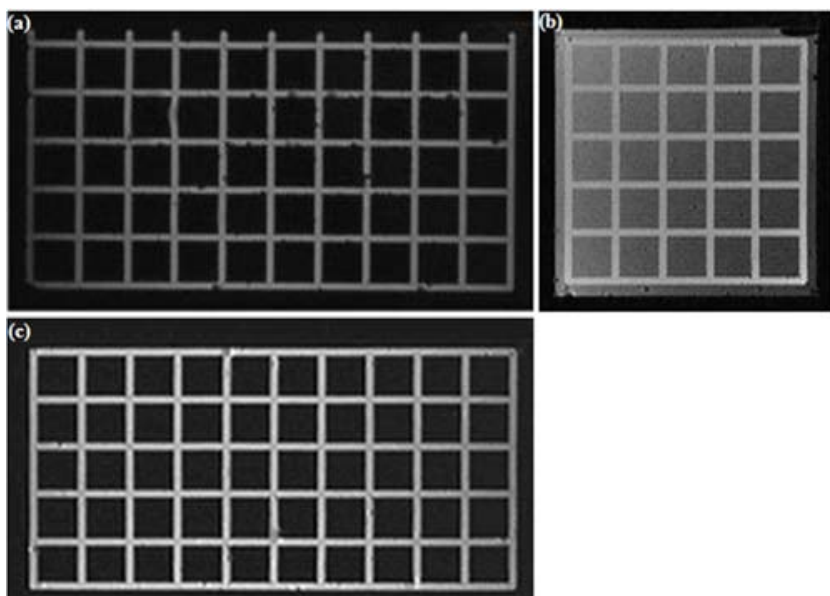

Figure 9. Spatial linearity (a: longitudinal, b: transversal, c: coronal).

MRI machine presents better SL because all values of distortion percentage are less than $5 \%$.

\subsection{Spatial Resolution (SR)}

Observation of the slice image of the level of the spatial resolution pattern (Figure 10) allows distinguishing holes until those of $0.8 \mathrm{~mm}$ diameter. It leads the spatial resolution (SR) to be $0.8 \mathrm{~mm}$.

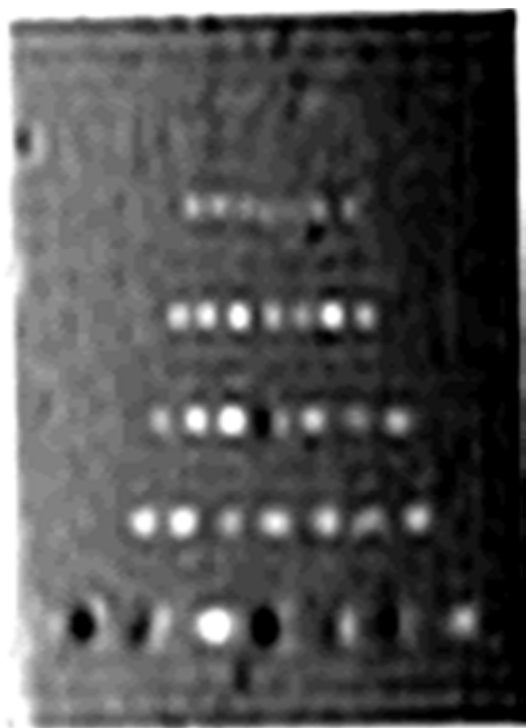

Figure 10. Spatial resolution.

\subsection{ST, Gap Separation and Position}

For the measurement of ST in the three spatial directions, we are interested by the acquisitions done at the levels of stairs ramps and star patterns (Figs 11-a and 11-b). The ST and gap are fixed on the MRI machine are respectively $3 \mathrm{~mm}$ and 6 $\mathrm{mm}$.

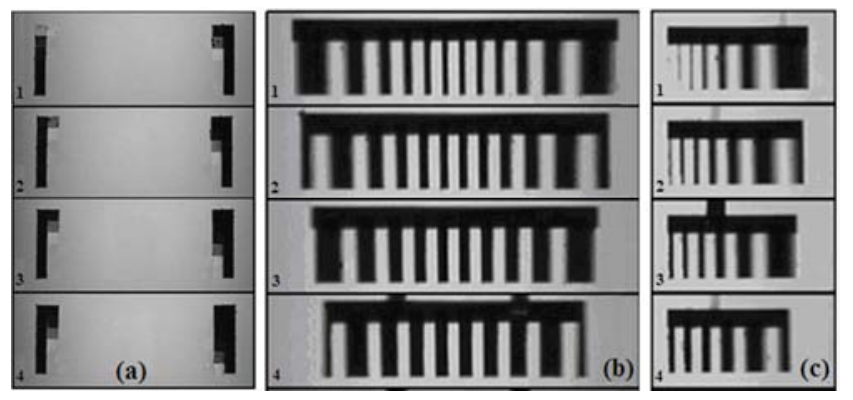

Figure 11. Measurement of ST (a: coronal, $b$ : longitudinal, $c$ : transversal).

For coronal acquisitions (Figure 11a), we can distinguish each part of images tree different tonalities of stairs. This means that the real ST is close to $3 \mathrm{~mm}$, which can also be verified and refined with the acquisition on linearity gradient with coronal acquisition.

For the longitudinal and transversal acquisitions (Figs 11-b and 11-c), we can see that the ST is in the vicinity of $3 \mathrm{~mm}$ for different slice positions. The observed fluctuations (Figure 12) do not reach the spatial resolution of the machine which shows better correlation between displayed and measured slice thickness.

Concerning slice gap, we can see on the curves of Figure 12 that the real value is nearby or equal to $6 \mathrm{~mm}$. We can also notice that the real slice positions almost coincide with fixed values and the fluctuations are less than the spatial resolution of the machine. 
(a)

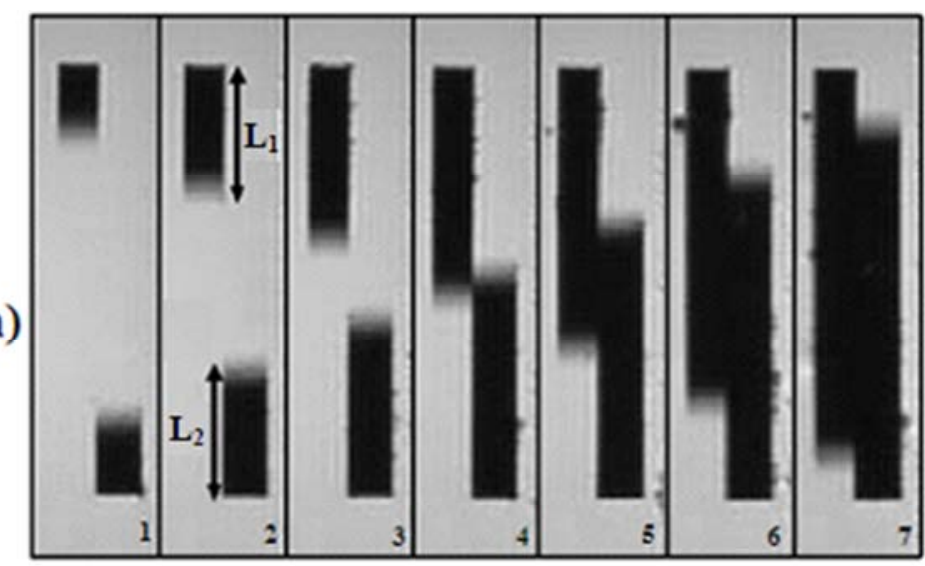

(b)

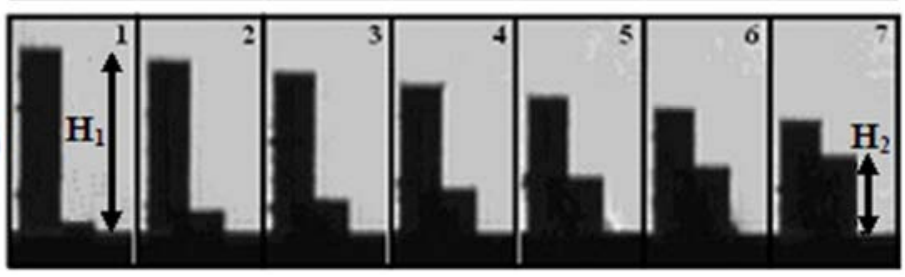

Figure 12. ST and position variations according to the rank of the slice (a: transversal acquisitions, $b$ : longitudinal acquisitions).

\subsection{Gradient Linearity (GL)}

The accuracy of ST and position relies on the gradient linearity. It is controlled by conducting the attaining at various levels of GL pattern ( $\mathrm{ST}=3 \mathrm{~mm}$, gap $=6 \mathrm{~mm}$ ) (see Figure 13).

(a)
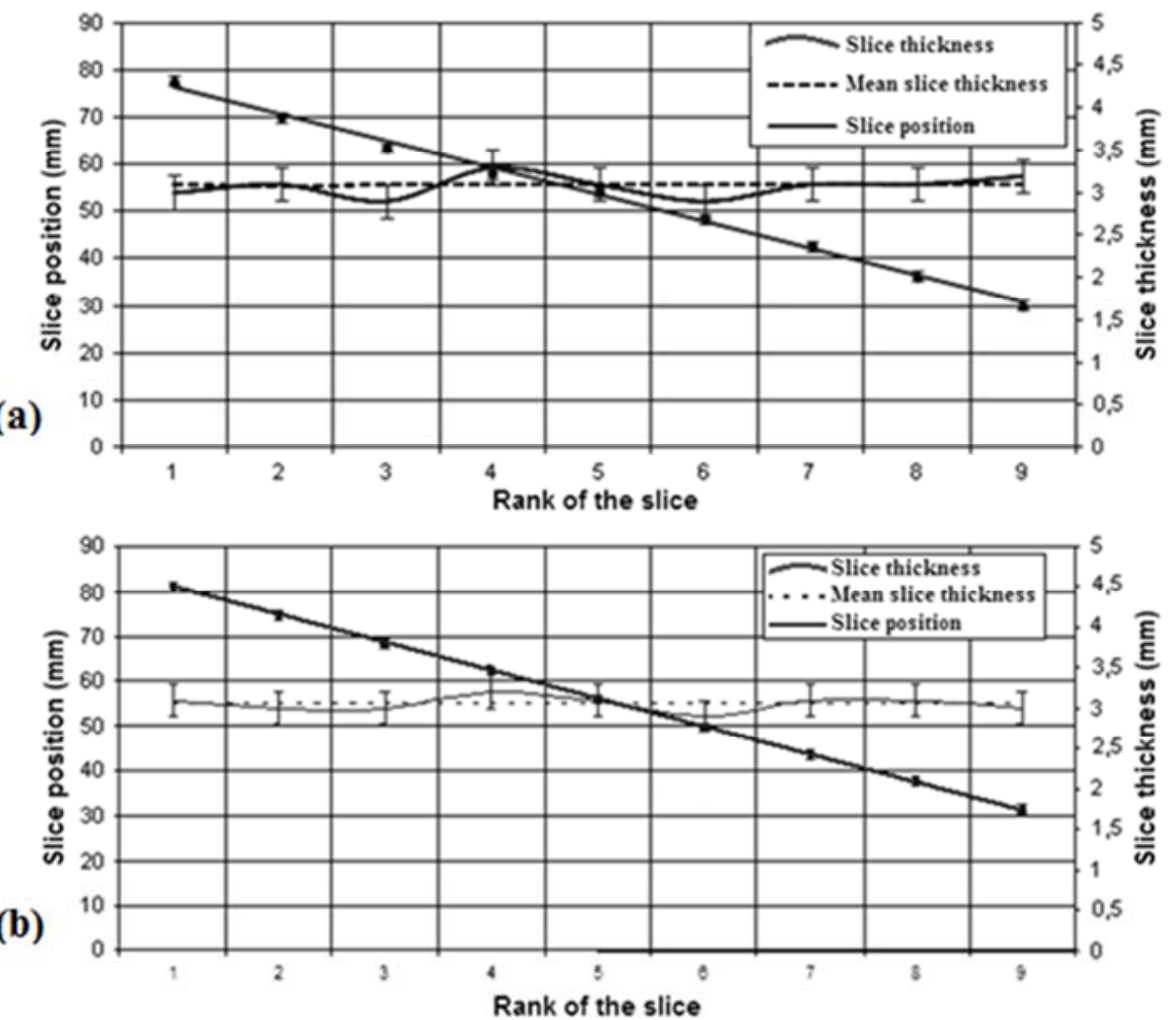

Figure 13. ST and position variations according to the rank of the slice (a: transversal acquisitions, b: longitudinal acquisitions).

If the field gradients are fully linear, the acquisitions with constant ST and gap at the level of the gradient field pattern (Figure 7-d) should give slice profiles whose lengths vary linearly in both directions. The slice profiles are of length $\mathrm{L}_{1}$ and L2 for coronal slices (Figure 14-a) and $\mathrm{H}_{1}$ and $\mathrm{H}_{2}$ for the transversal slices (Figure 14-b). The curves of Figure 14(a, b) show perfect linear variations $\left(\mathrm{R}^{2}=0.99\right)$ in these parameters which reflect linear field gradients. 

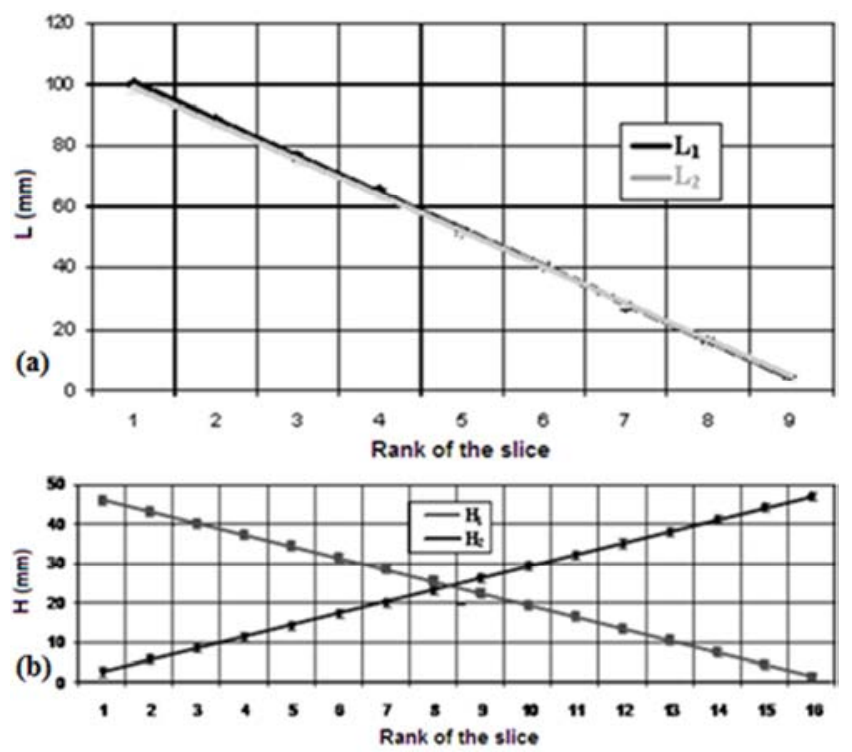

Figure 14. Slice profiles variations according to the rank of the slice ( $a: L_{i}$ and $L_{2}$ for coronal acquisitions, $b: H_{1}$ and $H_{2}$ for transversal acquisitions).

\subsection{Comparison of MTP with Other American College of Radiology Phantom}

American college of radiology phantom is considered the most used phantoms in the world in the field of MRI quality control [12]. It operates primarily in acrylic plastic and it costs $\$ 1716.00$.

The difference between the proposed MTP phantom and the ACR phantom lies in the easiness of use, competences, maintenance, materials used in fabrication, and quality to price ratio. First, it is easy to use because the positioning of the proposed MTP phantom in the MRI machine (Figure 15a) is easier and faster than the ACR phantom.

Indeed, for the MTP phantom, the position in a single direction can be checked that is achieved with a leveler, whereas the ACR phantom should be orientated so that the ramps of $45^{\circ}$ must be fully horizontal and variable inclined ramps must be vertical which is likely to be difficult to realize and sometimes requires the use of an accessory (Figure 15-b).

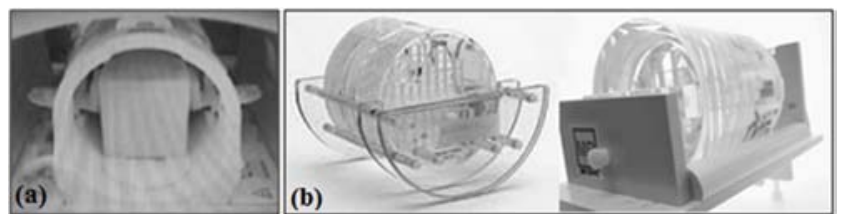

Figure 15. Positioning of the phantoms in the MRI antenna (a: without accessories for MTP phantom, $b$ : with accessories for ACR phantom).

Second, it is more competent because we can make sure that the tests of uniformity, $\mathrm{S} / \mathrm{N}$ and the central frequency $\mathrm{f}_{0}$ are made in the same way in a homogeneous region in the two phantoms. For the remaining parameters, we observe a clear advantage in terms of information provided for the proposed MTP phantom rather than from the ACR phantom. Indeed, for the exploration of geometric distortions if the test is performed only in a transversal position for the ACR phantom, it is realized in three directions for the phantom MTP (Figure 16).
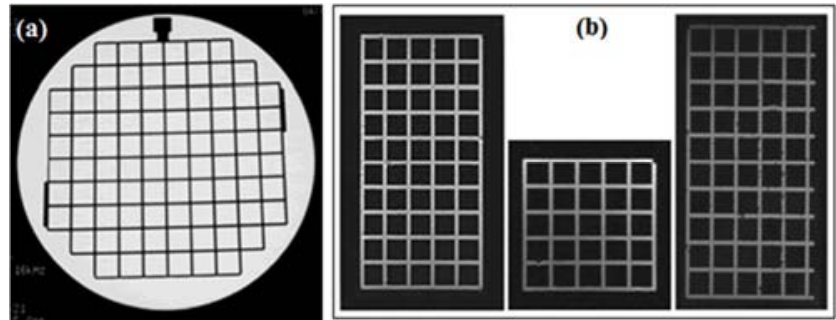

Figure 16. SL tests ( $a-A C R, b-F M T)$.

Whereas for the slice positions and thicknesses, they are generally made in one way of each direction for the ACR, but for the MTP phantom, it is always performed in two different ways for each direction (Figures 17 and 18). In addition, this allows us to assess the linearity of the three field gradients.

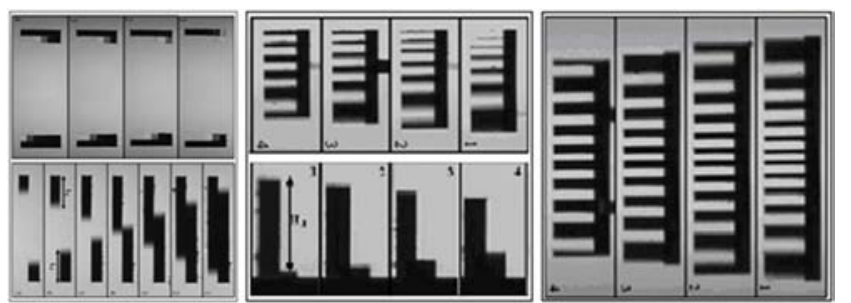

Figure 17. Slice positions and thickness measurement with the ACR phantom.

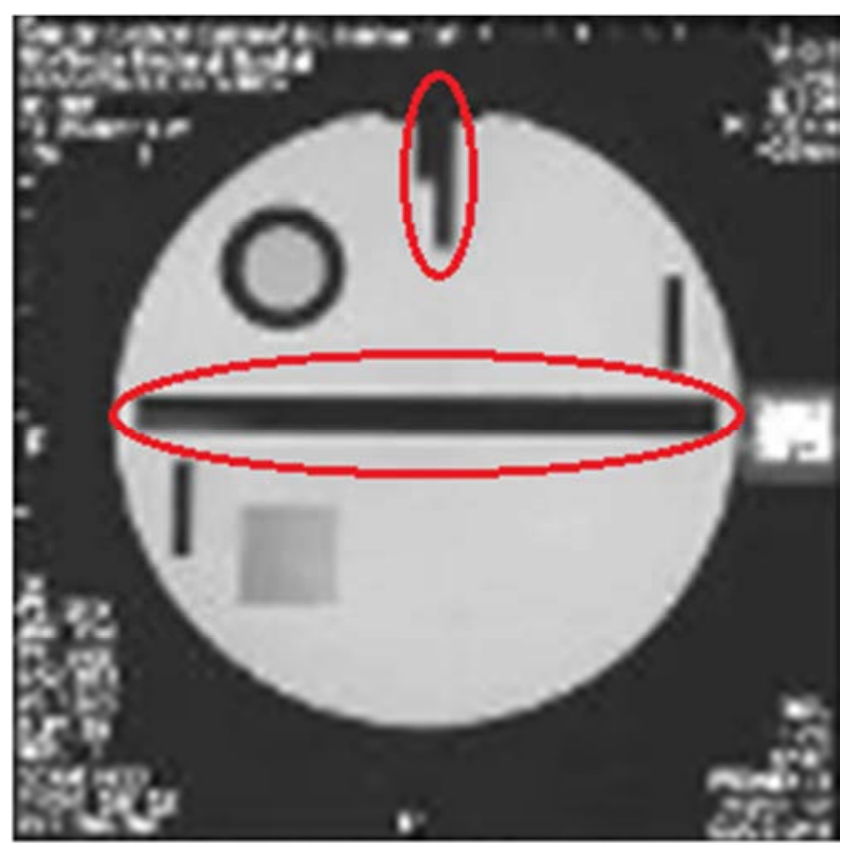

Figure 18. Slice positions and thickness measurement with the MTP phantom (from right to left: longitudinal, transversal and coronal).

Regarding the high contrast spatial resolution, the MTP phantom offers a wider range of measure $(0.5,0.8,1.0,1.3$, $1.5,2.0 \mathrm{~mm})$ than the ACR phantom $(0.9,1.0,1.1 \mathrm{~mm})$ which will be less accurate when testing the new MRI machines (Figure 19). 

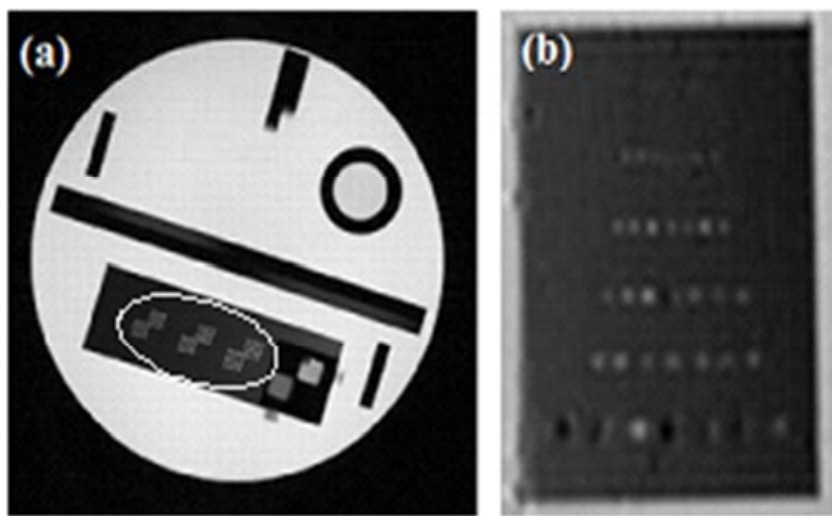

Figure 19. High contrast spatial resolution Tests ( $a$ - $A C R, b-M T P$ ).

Third, the maintenance of the MTP phantom is simpler as long as it is not hermetic like the ACR phantom. Thus, in case of failure of one component it is straightforward to open and to correct. We can still add components, such as a test pattern of low contrast delectability in all three directions. Such maneuvers are not possible when the ACR phantom is considered.

Regarding the production of phantoms, the ACR phantom is essentially made of acrylic material which is more expensive than polyamide of which we made the entire MTP. Establishing of MTP is cheaper and more accessible than ACR phantom. The realization and assembly of different components of the MTP needs at most two days of work. The price of our MTP cost only $\$ 400.00$, however, the cost of the ACR phantom is around $\$ 1716.00$. So, the MTP price cost nearly 0.2 times of ACR phantom. Also, for the level of quality of information of established MTP, It provided a very good quality/price ratio for our proposed MTP.

\section{Conclusions}

MTP phantom is proposed to investigate quality control and maintaining of MRI machine. It provides also much information on the operating status of the machine, and in case of default, it contributes to a definition of the effective root cause. The low cost and effectiveness compared to the ACR phantom may encourage practitioners to recommend it. The proposed MTP phantom is an effective solution especially in developing countries where we usually do not perform quality control testing in part because of the cost of necessary equipment (phantoms), which in term will increase diagnostic qualities of the images. The relatively large amount of information provided by the phantom urges us to achieve a platform of automatic stripping of information to facilitate the task of users. All in all, the developed MTP phantom offers more information of greater flexibility for the using and maintenance of magnetic resonance imaging.

\section{References}

[1] B. Kastler and D. Vetter, "Comprendre l'IRM: Manuel d'autoapprentissage," $7^{\text {th }}$ edition, 2011, ISBN: 978-2-294-71044-5.

[2] C. Chien-Chuan, W. Yung-Liang, W. Yau-Yau and L. HoLing, "Quality Assurance of Clinical MRI Scanners Using ACR MRI Phantom: Preliminary Results," J. of Digital Imaging, vol. 17 (4), pp. 279-284, 2004.

[3] R. R. Price, L. Axel, T. Morgan, R. Newman, W. Perman, N. Schneiders, M. Selikson, M. L. Wood and S. R. Thomas, "Quality assurance methods and phantoms for magnetic resonance imaging Task Group No. 1.", DOI:10.1118/1.596566.

[4] S. N. Sotiropoulos, S. Jbabdi, J. Xu, J. L. Andersson, S. Moeller, E. J. Auerbach, M. F. Glasser, M., G. Hernandez Sapiro, M. Jenkinson, D. A. Feinberg, E. Yacoub, C. Lenglet, D. C. Van Essen, K. Ugurbil andT. E. Behrens, "Advances in diffusion MRI acquisition and processing in the Human Connectome Project" Minn HCP Consortium, Neuroimage, vol. 80, pp. 125-43, 2013.

[5] R. R. Price, L. Axel, T. Morgan, R. Newman, W. Perman, N. Schneiders, M. Selikson, M. L. Wood and S. R. Thomas, "Quality assurance methods and phantoms for magnetic resonance imaging," Med. Phys., vol. 17, N. 2, pp. 287-95, 1990.

[6] R. Larsen, M. Nielsen and J. Sporring, "Automated analysis of multi-site MRI phantom data for the NIHPD Project," LNCS 4191, pp. 144-151, Springer-Verlag Berlin Heidelberg, MICCAI 2006.

[7] P. Colomboa, A. Baldassarri, M. Del Corona, L. Mascaro and S. Strocchi, "Multicenter trial for the set-up of a MRI quality assurance programme," Magn. Res. Imag., vol. 22, pp. 93$101,2004$.

[8] B. R. Condon, J. Patterson and D. Wyper, "Image non uniformity in magnetic resonance imaging: its magnitude and methods for correction,” Br. J. Radiol., vol. 60, pp.83-87, 1987.

[9] Q. Bao, F. Chen, L. Chen, K. Song, Z. Liu and C. Liu, “A new gradient shimming method based on undistorted field map of $\mathrm{B}_{0}$ inhomogeneity," J. of Mag. Res., vol. 265, pp. 25-32, 2016.

[10] D. W. McRobbie, R. A. Lerski and K. Straughan, "Investigation of slice characteristics in nuclear magnetic resonance imaging," Phys. Med. Biol., vol. 31, pp. 613-626, 1986.

[11] M. Selikson and T. Fearon, "Averaging error in NMR slice profile measurements," Magn. Res. Med., vol. 7, pp. 280-284, 1988.

[12] K. A. Kraft, P. P. Fatouros, G. D. Clarke and P. Kishore, "An MRI phantom material for quantitative relaxometry," Mag. Res. Med., vol. 5, pp. 555-562, 1987.

[13] P. Colomboa, A. Baldassarri, M. Del Corona, L. Mascaro and S. Strocchi, "Multicenter trial for the set-up of a MRI quality assurance programme"; Mag. Res. Imag., vol. 22, pp. 93-101, 2004. 